\title{
Molecular characterisation of breast cancer patients at high and low recurrence risk
}

\author{
Serena Bonin • Davide Brunetti • Elena Benedetti • \\ Isabella Dotti • Nader Gorji • Giorgio Stanta
}

Received: 23 August 2007 / Accepted: 20 December 2007 / Published online: 31 January 2008

(C) Springer-Verlag 2008

\begin{abstract}
The ability to predict the recurrence risk in breast cancer patients is not available for the individual. It is commonly accepted that the different clinical course of tumours with identical histology and stage are the result of differences at the molecular level. This case study of 80 patients affected by breast cancer looked at the messenger ribonucleic acid expression level of 22 genes, by using quantitative reverse transcriptase-polymerase chain reaction. Our results showed that a panel of seven genes is associated to patients' survival. Moreover, the combination of two couples of genes is able to define short- and longliving cohorts of patients. In particular, our findings strongly demonstrate that retinoblastoma (RB) and cyclin-
\end{abstract}

\footnotetext{
S. Bonin $\cdot$ I. Dotti $\cdot$ G. Stanta

Department of Clinical,

Morphological and Technological Sciences,

International Centre for Genetic Engineering and Biotechnology,

University of Trieste,

Trieste, Italy

D. Brunetti

Friuli-Venezia Giulia Cancer Registry,

Udine, Italy

E. Benedetti

International Centre for Genetic Engineering and Biotechnology,

Trieste, Italy

N. Gorji

Surgical Pathology Unit, S. Andrea Hospital,

La Spezia, Italy

G. Stanta $(\bowtie)$

Surgical Pathology Unit, Cattinara Hospital,

Strada di Fiume 447,

34149 Trieste, Italy

e-mail: stanta@icgeb.org
}

dependent kinase 2 (CDK2) on one side and cytokeratin 8 (CK8) and epidermal growth factor receptor 2 (HER2) on the other may affect the clinical course of the disease in $56 \%$ of patients. Groups characterised by low RB and high CDK2 as with low CK 8 and high HER2 have a higher risk of recurrences and death in 5 years. The identification of these sub-groups of patients with higher risk of early relapse could have further involvement in the selection of the cases to submit to therapy against HER2 or CDK2 as a possible therapy target.

Keywords Breast cancer - Formalin-fixed paraffinembedded tissues · Quantitative RT-PCR · RB . $\mathrm{CDK} 2 \cdot \mathrm{HER} 2 \cdot \mathrm{CK} 8$

\section{Introduction}

Breast cancer is the leading cause of death among women in developed countries [30]. Despite important advances in therapy, more than half of the affected patients undergoes relapses [17].

Many studies on gene expression carried out by microarrays have identified expression profiles and gene sets that are prognostic and/or predictive of outcome of breast cancer patients. Unfortunately, genes identified by these studies show only a slight overlap, even if they seem to be able to predict the outcome of the same group of patients [7]. The major concern related to the use of microarray platforms is the requirement of high-quality ribonucleic acid (RNA), generally derived from frozen samples. Such tumour sets are often small, and an extensive clinical history is not yet available, whereas formalin-fixed paraffin-embedded (FFPE) materials are abundant and allow the possibility of selecting samples related to patients that have been 
followed-up for long periods. Although reverse transcriptasepolymerase chain reaction (RT-PCR)-based methods from FFPE samples can have clinical application, genome-wide microarray analysis has proven to be difficult. A recent paper by Penland et al. [19], has demonstrated that only a minority of FFPE blocks could be analysed by microarray.

To investigate the progression of breast carcinoma, we analysed the level of expression of a well-defined panel of genes involved in the maintenance/abrogation of the cell senescence machinery in primary breast cancers. This study represents an extension of a previous paper where we analysed a part of this cohort of patients for cell cyclerelated molecules, such as cyclins, cyclin-dependent kinases $(\mathrm{CDKs})$ and the phosphatase cell division cycle 25a (CDC25a) [2].

Recent studies have demonstrated that the first step in creating an in vitro model of human cancer involves the abrogation of the cellular senescence mechanism. In fact, a tumour will develop only if it has bypassed all senescence controls [5]. Two tumour suppressor genes, retinoblastoma and $\mathrm{p} 53$, are central factors in replicative and premature senescence. Suppression or down-regulation of the retinoblastoma pathway together with the activation of immortalisation factors, such as telomerase activation and RAS pathway activation, is essential to define breast cancer tumourigenesis and progression [12], also at a clinical level. By using quantitative RT-PCR, we looked at the messenger
RNA (mRNA) expression level of 22 genes belonging to the RAS pathway (epidermal growth factor receptor 1 [EGFr1] and 2 [HER2], k-RAS, v-raf-1 murine leukaemia viral oncogene homolog 1 [RAF-1], mitogen-activated protein kinase 1 [ERK1], phosphatidylinositol 3 kinase [PI3K], protein kinase $\mathrm{B}$ isoform 3 [AKT3]), cell cycle regulation/ senescence (Cyclin A, D1 and E1, CDK2, CDK4, CDK6, CDK inhibitor 2A [p16], retinoblastoma 2 [p130-RB2], retinoblastoma [RB], CDC25a), telomerase complex (mRNA coding for catalytic component and RNA component, hTRT and hTR, respectively) and differentiation/metastasis suppression genes such as non-metastatic cells 1 protein (NM23), sel1 suppressor of lin-12-like (SEL-1L) and cytokeratin 8 (CK8). Genes were selected according to their known links to malignant cell behaviour in breast cancer and their importance in major cancer hallmarks, such as cell senescence/proliferation and survival. Our main goal was to investigate whether the gene expression profiles of the reported molecular markers in primary tumours are predictive of metastatic potential.

\section{Materials and methods}

Eighty women were selected who were residents in the Northeastern Italian province of Trieste and whose first primary cancer was unilateral infiltrating duct breast carcinoma. Cancers were diagnosed at the age of 34 or older,
Table 1 Oligonucleotide sequences

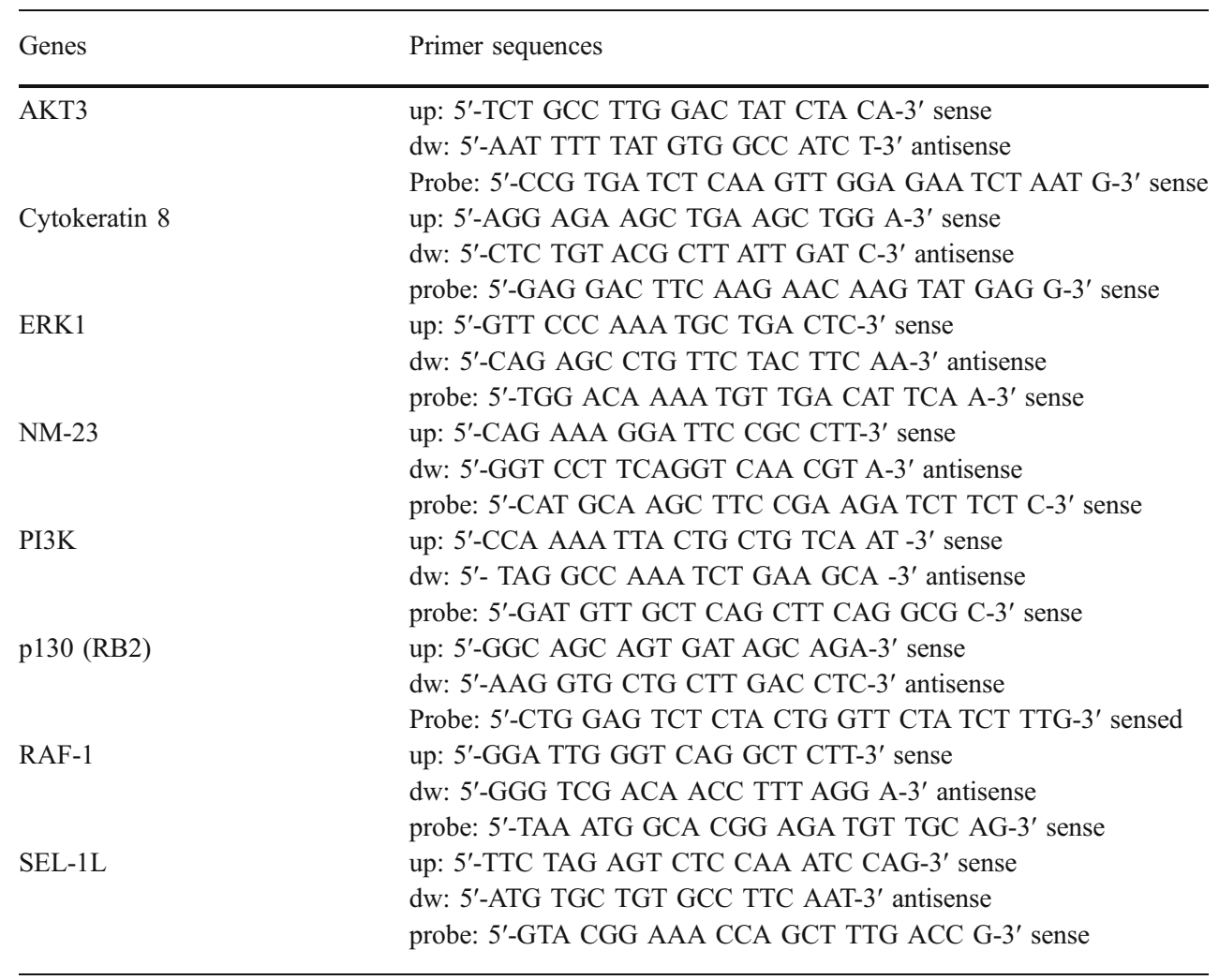

Primers up and down (dw) were used in the PCR reaction. The probe was used in the hybridisation of the dot blots. 
Table 2 RT-PCR and hybridisation conditions

\begin{tabular}{|c|c|c|c|c|c|}
\hline \multirow[t]{2}{*}{ Marker } & \multicolumn{3}{|c|}{ PCR conditions } & \multicolumn{2}{|c|}{$\begin{array}{l}\text { Hybridisation } \\
\text { conditions }\end{array}$} \\
\hline & $\begin{array}{l}\mathrm{RNA}^{\mathrm{a}} \\
\text { (ng) }\end{array}$ & $T_{\mathrm{a}}^{\mathrm{b}}\left({ }^{\circ} \mathrm{C}\right)$ & PCR cycles ${ }^{\mathrm{c}}$ & $T^{\mathrm{d}}\left({ }^{\circ} \mathrm{C}\right)$ & $\begin{array}{l}\text { Formamide } \\
\%^{\mathrm{e}}\end{array}$ \\
\hline AKT3 & 125 & 50 & 45 & 52 & 3.0 \\
\hline Cytokeratin 8 & 500 & 50 & 50 & 46 & 0.0 \\
\hline ERK1 & 30 & 50 & 35 & 52 & 3.0 \\
\hline NM23 & 100 & 50 & 40 & 50 & 0.0 \\
\hline PI3K & 60 & 50 & 30 & 52 & 8.0 \\
\hline p130 (RB2) & 250 & 55 & 55 & 45 & 0.0 \\
\hline RAF-1 & 120 & 55 & 40 & 52 & 4.2 \\
\hline SEL-1L & 15 & 51 & 45 & 45 & 0.0 \\
\hline
\end{tabular}

${ }^{\text {a }}$ Quantity of total RNA used in the cDNA synthesis

${ }^{\mathrm{b}}$ Annealing temperature for PCR reaction

${ }^{\mathrm{c}}$ Number of PCR cycles

${ }^{\mathrm{d}}$ Hybridisation Temperature

${ }^{\mathrm{e}}$ Percentage of Formamide included in the hybridisation solution

between 1971 and 1989 and treated only with Halsted radical mastectomy and complete axillary dissection, without any additional chemotherapy or radiotherapy. Seventy-three patients belonging to this cohort were also part of a previous study [2]. All the slides from the primary tumour were reviewed by one of the authors (Stanta). Carcinomas were histologically graded according to Elston and Ellis [6] and grouped in stages according to the TNM Classification of Malignant Tumours, 6th edition [10].

The cohort of women was followed through the general population-based Friuli-Venezia Giulia Cancer Registry [1] from diagnosis of breast cancer to death or until 31 December 2006, whichever came first [18]. One patient was lost in 1993, after 4 years of follow-up. During the observation period, 69 women died, and all of them underwent complete autopsy at the Surgical Pathology Department of the University of Trieste.

FFPE specimens from primary breast cancers were microdissected and used to extract and quantify the RNA as described in detail elsewhere [3, 24, 27]. RT-PCR was performed as previously reported [24]. Cyclins A, D1 and E, CDK2, 4 and 6 and CDC25a were previously analysed in 73 patients [2] and seven additional patients. Oligonucleotides and RT-PCR conditions were already described for: cyclins A, D1 and E, CDK2, 4 and 6 and CDC25a [2], p16, RB, k-RAS, EGFr1, HER2 [25] and telomerase component genes [26]. For AKT3, CK8, ERK1, NM23, RAF-1, PI3K and SEL-1L and p130 (RB2) oligonucleotides, PCR and hybridisation conditions are reported in Tables 1 and 2 . We used paraffin blocks without tissues as negative controls. The basal level of expression of the genes was quantified by analysing ten normal mammary glands, from biopsies negative for cancer performed in the same range of time as the cases. The baseline expression level was therefore detected for epithelial, myoepithelial and fibrous tissue but not adipose tissue elements of the normal gland. RNA extracted from the breast cancer cell line (BT-549) was used as a positive control. To test the reproducibility of the method, for every gene analysed, we randomly selected ten samples from the case study for re-submission for RTPCR analyses. Amplification products were quantified by dot hybridisation as previously reported [24]. Standardisation using housekeeping genes was reported in detail elsewhere [2, 3].

$T$ test analysis was applied to compare the mean value of expression of each gene in patients with recurrences and in those without metastasis. To estimate the joint effects of the analysed covariates on patients' survival, the data were analysed by fitting the Cox proportional hazard regression model as previously reported [2]. Cox proportional hazard analysis included age at diagnosis, stage and grade of the tumours and the complete set of the molecular markers. In this analysis, tumours with expression of molecular markers lower or higher than the mean value were classified as low or high status of expression, respectively.

To investigate possible associations among the genes detected by Cox regression analysis, we chose pairs of molecular markers involved in opposite or conflicting functions. We considered groups defined by: high expression status of one marker and low expression status of the other marker as well as the opposite situation. The chosen pairs of genes were: HER2 and CK8 as de-differentiation/ differentiation markers, $\mathrm{CDK} 2$ and $\mathrm{RB}$ as markers of cell cycle progression/senescence, PI3K-RAF1 and AKT3RAF1 as markers of the main branches of the RAS pathways and PI3K and AKT3 as markers of activation of cell transcription factors. The log-rank test was used to compare the survival between the above mentioned groups.

Statistical analyses were performed with the package Stata/SE 9.0 (Stata, College Station, TX).

Table 3 Characteristics of the 80 breast carcinomas by stage, grading and patients' age at diagnosis

\begin{tabular}{lllllll}
\hline \multirow{2}{*}{ Stage } & \multicolumn{2}{c}{ Grading $^{\mathrm{a}}$} & & \multirow{2}{*}{ Number of cases } & \multicolumn{2}{c}{ Age at diagnosis (years) } \\
\cline { 2 - 3 } & G1 & G2 & G3 & & Mean & Range \\
\hline I & 14 & 15 & 4 & 33 & 65 & $38-79$ \\
IIA & 7 & 14 & 5 & 26 & 63 & $35-87$ \\
IIB & 1 & 4 & 1 & 6 & 56 & $48-63$ \\
IIIA & 3 & 6 & 2 & 11 & 57 & $34-76$ \\
IIIC & 0 & 3 & 1 & 4 & 70 & $47-90$ \\
All stages & 25 & 42 & 13 & 80 & 61 & $34-90$
\end{tabular}

${ }^{a} G 1$ Well differentiated, G2 moderately differentiated, G3 poorly differentiated 
Table 4 Results of Cox multivariate analysis

\begin{tabular}{|c|c|c|}
\hline Variables & Hazard ratio $(p)^{\mathrm{a}}$ & $95 \% \mathrm{CI}^{\mathrm{b}}$ \\
\hline $\mathrm{CDK} 2$ & $5.3(0.04)$ & $1.1-25.4$ \\
\hline HER2 & $3.8(0.02)$ & $1.2-12.2$ \\
\hline PI3K & $3.2(0.06)$ & $0.9-10.8$ \\
\hline CYCLIN E & 2.4 & $0.8-6.5$ \\
\hline RAF1 & $2.3(0.05)$ & $1.0-5.6$ \\
\hline ERK1 & 2.0 & $0.8-4.9$ \\
\hline CDK4 & 1.8 & $0.4-9.1$ \\
\hline STAGE & 1.5 & $0.9-2.5$ \\
\hline HTR & 1.5 & $0.6-3.5$ \\
\hline CYCLIN A & 1.5 & $0.7-3.0$ \\
\hline $\mathrm{CDC} 25 \mathrm{a}$ & 1.4 & $0.3-7.5$ \\
\hline CDK6 & 1.3 & $0.5-3.3$ \\
\hline EGFR1 & 1.1 & $0.5-2.4$ \\
\hline SEL1L & 1.1 & $0.5-2.8$ \\
\hline CYCLIN D1 & 1.0 & $0.3-3.5$ \\
\hline P16 & 1.0 & $0.5-2.1$ \\
\hline AGE & 1.0 & $0.9-1.0$ \\
\hline $\mathrm{RB} 2$ & 0.8 & $0.3-2.4$ \\
\hline GRADE & 0.7 & $0.3-1.4$ \\
\hline NM23 & 0.6 & $0.2-2.1$ \\
\hline K-RAS & 0.6 & $0.3-1.2$ \\
\hline HTRT & 0.6 & $0.2-1.4$ \\
\hline $\mathrm{RB}$ & $0.3(0.03)$ & $0.1-0.9$ \\
\hline CK8 & $0.3(0.04)$ & $0.1-1.0$ \\
\hline AKT3 & $0.2(0.01)$ & $0.09-0.7$ \\
\hline
\end{tabular}

${ }^{\text {a }}$ Probability value is reported only for markers that affected significantly patients' survival.

${ }^{\mathrm{b}}$ Confidence interval

\section{Results}

The cohort of 80 women affected by breast cancer showed a median age at diagnosis of 61 years $(25 \mathrm{th}-75$ th percentile $=$ 51-70). All of them were affected by infiltrating duct carcinoma of the breast, the diameter of which was not larger than $3.0 \mathrm{~cm}$ at pathological examination. Staging and grading of tumours are shown in Table 3. The axillary lymph nodes were tumour negative in 43 patients, while they were positive in 37 patients at the time of diagnosis of the primary tumour. The median number of positive nodes was 3 (range $=1-15$ ). During the period of observation, 42 patients developed distant metastases.

The median duration of follow-up was 6 years $(25$ th75 th percentile $=3-12$ years). Of the 69 deceased patients, 42 died of breast cancer metastases, one patient died of ovary carcinoma and 26 of non-neoplastic diseases.

This study confirms our previous findings on CDC25a $(p=0.01), \mathrm{CDK} 2(p=0.01)$ and $\mathrm{CDK} 4(p=0.03)$ expression in the groups of patients who developed recurrences and the patients that did not [2]. Moreover, the present study also confirms that CDK2 and CDC25a affected patients' survival in univariate analysis ( $p=0.0001$ and $p=0.02$, respectively) as previously reported [2].

The results of the Cox proportional hazard regression analysis are reported in detail in Table 4. An independent influence on cancer-specific death was detected for AKT3 $(p=0.01), \mathrm{RAF}-1 \quad(p=0.05), \mathrm{CDK} 2(p=0.04), \mathrm{CK} 8(p=$ $0.04), \operatorname{RB}(p=0.03)$ and HER2 $(p=0.02)$. A borderline influence on patients' survival was detected for PI3K ( $p=$ 0.06). Conversely, tumour stage and grade, patients' age at diagnosis and the remaining molecular markers analysed did not show an effect on survival. After dividing the stage into lymph node status (positive or negative for metastasis) and tumour dimension (diameter expressed in centimetres), the results of Cox proportional hazard regression analysis were restated. In this analysis, lymph node status and tumour diameter did not affect patients' survival $(p=0.1$ and $p=0.5$, respectively).
Fig. 1 Survival curves related to Cox proportional hazard regression with respect to AKT3 expression level in the subgroups of low AKT3 (AKT3 expression lower than the mean value) or high AKT3 (AKT3 expression higher than the mean value)

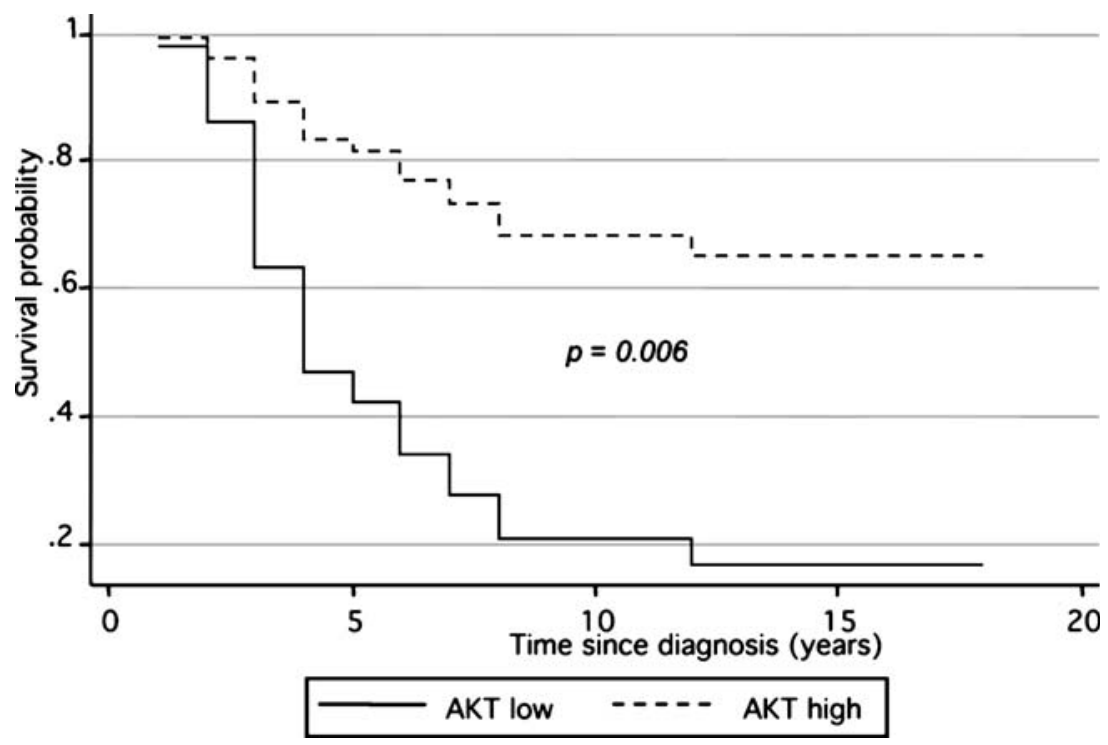


Fig. 2 Survival curves related to Cox proportional hazard regression with respect to cytokeratin 8 expression level in the sub-groups of low cytokeratin 8 (cytokeratin 8 expression lower than the mean value) or high cytokeratin 8 (cytokeratin 8 expression higher than the mean value)

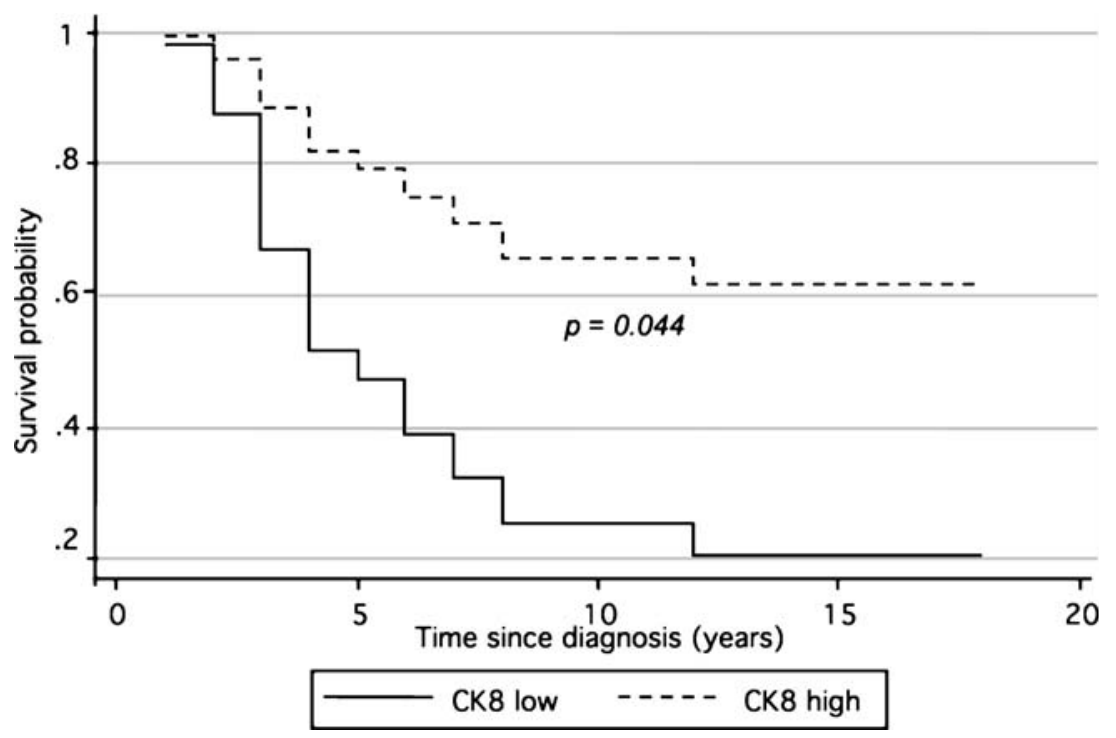

Among the genes related to survival, AKT3, CK8 and RB seem to have a protective effect. Higher expression levels were indeed associated with better prognosis (Figs. 1, 2 and 3, respectively). Conversely, CDK2, HER2, PI3K and RAF-1 showed a worse effect on patient survival (Figs. 4, 5, 6 and 7, respectively). In particular, CDK2 and HER2 high-expression statuses seem to be associated with early relapses, and patients' survival decreased to 27 and $36 \%$ at 5 years, respectively (Figs. 4 and 5).

Moreover, groups of patients with a high-expression status of RB but a low-expression status of CDK2 showed a longer disease-free survival in comparison to the group characterised by low RB status and high CDK2 status ( $p=$ $0.001)$ as described in Fig. 8. A borderline significance ( $p=$ 0.07) was detected when pairing CK 8 and HER 2 statuses (Fig. 9). The associations RAF1-PI3K and PI3K-AKT3 as well as the pairing between RAF1 and AKT3 did not affect patients' survival ( $p=0.9$ and $p=0.7$, respectively).

Seventy-three percent of patients belonging to the group with low RB and high CDK2 statuses died during the first 5 years of follow-up. A similar pattern was found for the group described by low CK8 and high HER2, in which $47 \%$ of patients died within the fifth year of follow-up. Moreover, the groups described by these two couples did not share any patient. Conversely, breast cancer patients described by high RB and low CDK2 showed a 5- and a 10 -year survival probability of 77 and $68 \%$, respectively. Similarly, the survival probability of patients with high CK8 and low HER 2 was 81 and $69 \%$ at 5 and 10 years, respectively. These two groups overlapped for three patients. The four groups identified by these couples of markers included 45 of the 80 patients $(56 \%)$.
Fig. 3 Survival curves related to Cox proportional hazard regression with respect to retinoblastoma expression level in the sub-groups of low retinoblastoma (RB expression lower than the mean value) or high retinoblastoma $(\mathrm{RB}$ expression higher than the mean value)

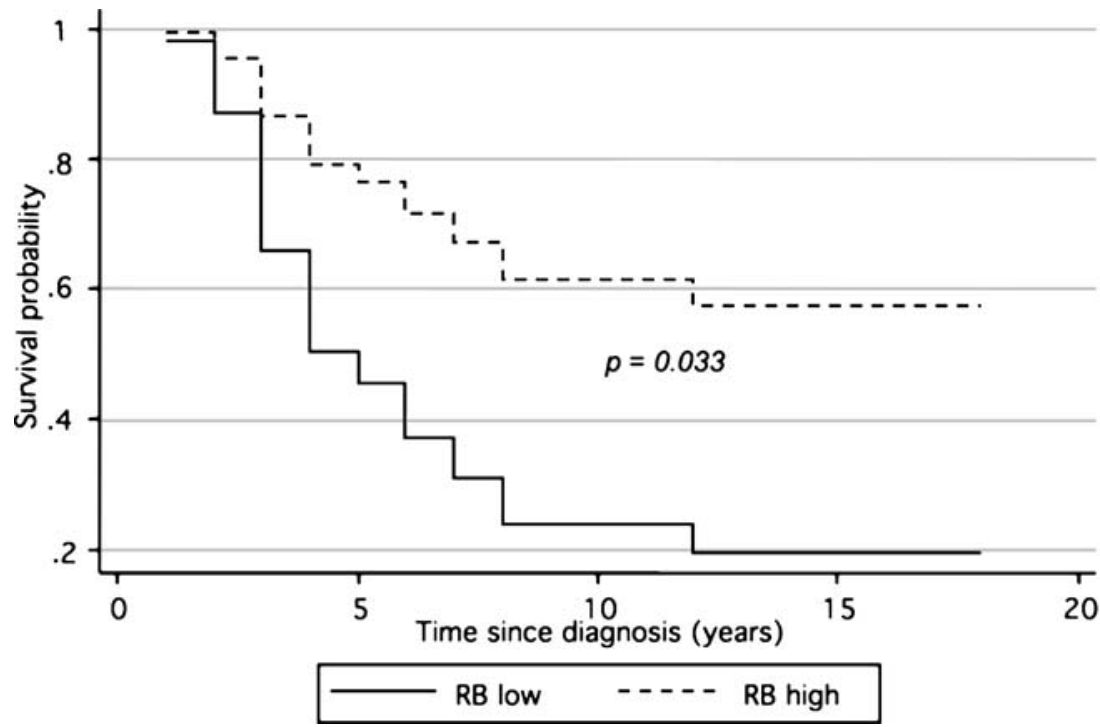


Fig. 4 Survival curves related to Cox proportional hazard regression with respect to $\mathrm{CDK} 2$ expression level in the subgroups of low CDK2 (CDK2 expression lower than the mean value) or high CDK2 (CDK2 expression higher than the mean value)

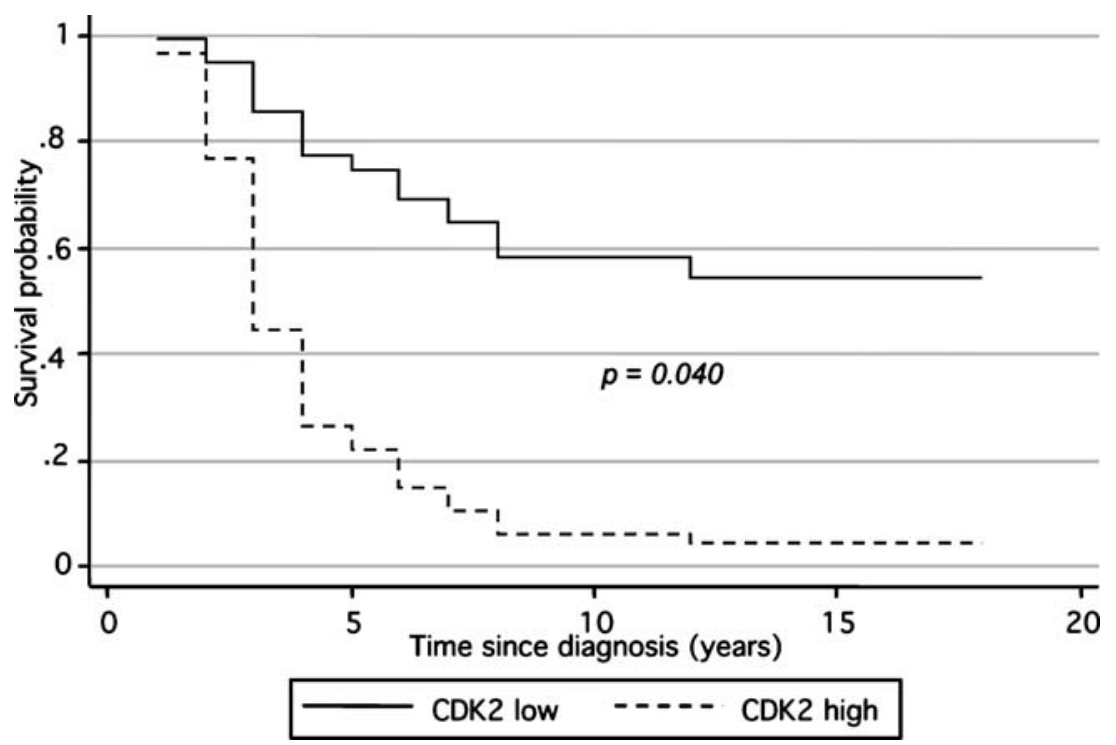

\section{Discussion}

The ability to define the risk of recurrence is useful in medical decision making. Several clinical and pathological factors have been proposed to predict the recurrence of breast cancer. However, at the present time, it is not possible to predict recurrence vs no recurrence at an individual level. It is commonly accepted that the different clinical courses of patients characterised by the same histology and stage are the result of molecular differences among cancers [21]. Different single molecular markers have been assessed in the past 20 years but failed to be considered for clinical use for various reasons [21]. Microarray studies have identified expression profiles and gene sets that are prognostic or predictive or both for patients with breast cancer. Comparison of the lists of genes derived from these studies shows only a slight overlap probably because of differences in the cohorts of patients, microarray platforms and mathematical methods of analysis [7]. Understanding the molecular alterations connected with progression to eventual recurrence is of particular importance, especially for therapeutic decisions.

In the present study, carried out on 80 women with infiltrating duct carcinoma of the breast, quantitative RTPCR was performed for a set of 22 genes involved in cell senescence, malignant transformation and tumour suppression. This study confirms our previous findings on the expression level of CDK2, CDK4 and CDC25a in breast cancer progression [2] and their correlation with recurrences. As previously reported [2], CDK2 over-expression seems to have an influence on the patient's survival both by uni- and multi-variate analysis, while data on CDC25a and
Fig. 5 Survival curves related to Cox proportional hazard regression with respect to HER2 expression level in the subgroups of low HER2 (HER2 expression lower than the mean value) or high HER2 (HER2 expression higher than the mean value)

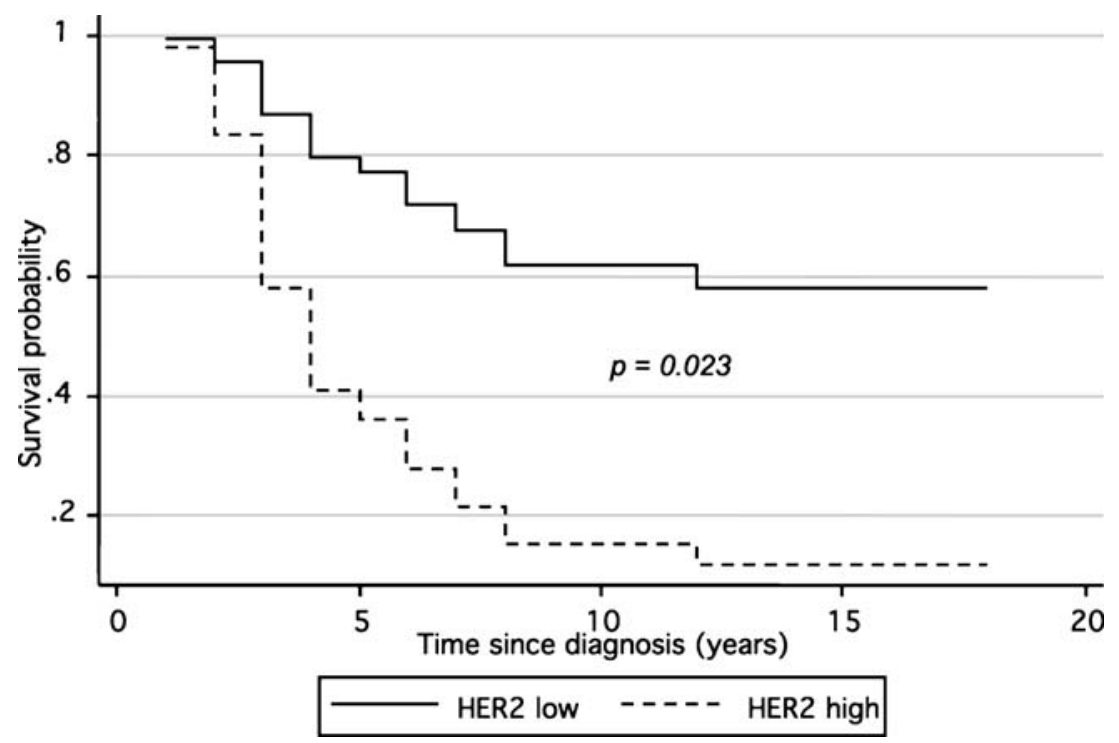


Fig. 6 Survival curves related to Cox proportional hazard regression with respect to $\mathrm{PI} 3 \mathrm{~K}$ expression level in the subgroups of low PI3K (PI3K expression lower than the mean value) or high PI3K (PI3K expression higher than the mean value)

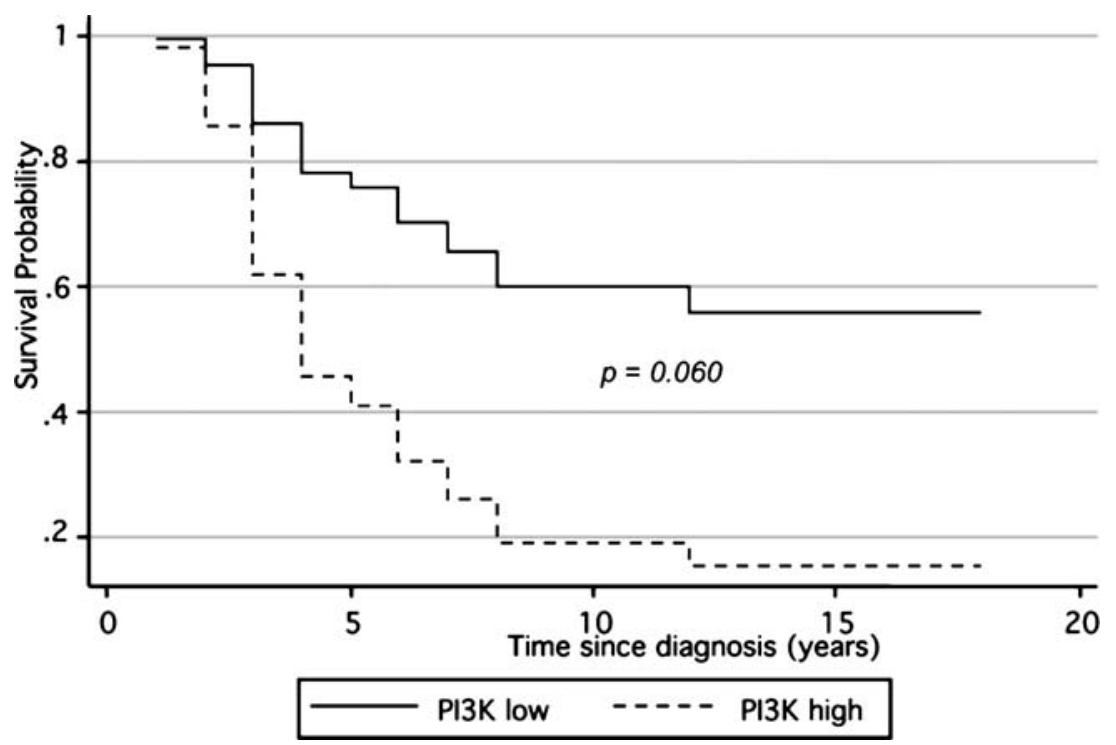

survival were confirmed only by uni-variate analysis. In our study, Cox proportional hazard analysis did not identify tumour stage as significantly affecting survival in these patients $(p=0.1)$. The main differences between the previous and the present study is the enrolment of seven women in the cohort of patients and 15 new molecular markers that have been compared with the previous ones.

Patients' survival was analysed by the Cox proportional hazard regression method. We divided the cases with a high level of expression (over the average value) from those with lower expression (below the average value). In this analysis, only those genes that showed a significant level of survival between the two groups are discussed.

Cox proportional hazard analysis identified different molecules involved in patients' survival. In particular, our results showed that patients with a higher expression level of HER2 and/or PI3K and a lower expression level of AKT seem to have the worst outcome. Our data agree with previous reports. It is well known that alteration in the $\mathrm{PI} 3 \mathrm{~K} / \mathrm{AKT}$ signalling cascade is common in human cancers and results in hyper-activation of the pathway that leads to tumour progression [8]. In particular, our data associate a worse outcome to patients over-expressing HER2 and/or PI3K but not AKT. The association of higher AKT3 mRNA expression level with better prognosis shown in our study is discordant with a previous paper [29]. A possible explanation of this discrepancy is related to methodological issues, as Totunaka et al. detected AKT in its activated status, i.e. the expression of phosphorylated AKT by immunohistochemistry (IHC). Our primer set was able to identify only isoform 3 of the transcript (AKT3). In fact recently, Maroulakou et al. reported that AKT2 ablation accelerates
Fig. 7 Survival curves related to Cox proportional hazard regression with respect to RAF1 expression level in the subgroups of low RAF-1 (RAF-1 expression lower than the mean value) or high RAF-1 (RAF-1 expression higher than the mean value)

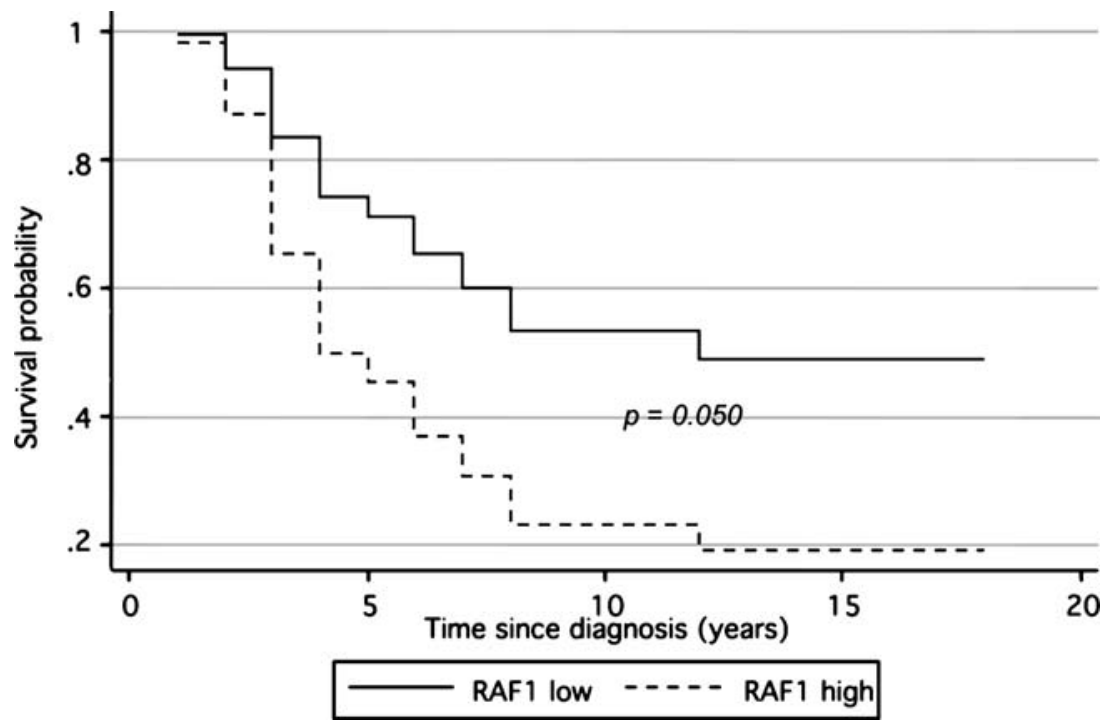


Fig. 8 Kaplan-Meier survival curves for patients with respect to $\mathrm{RB}$ and $\mathrm{CDK} 2$ expression level in the sub-groups of high $\mathrm{RB}$ and low CDK2 or low RB and high CDK2 status

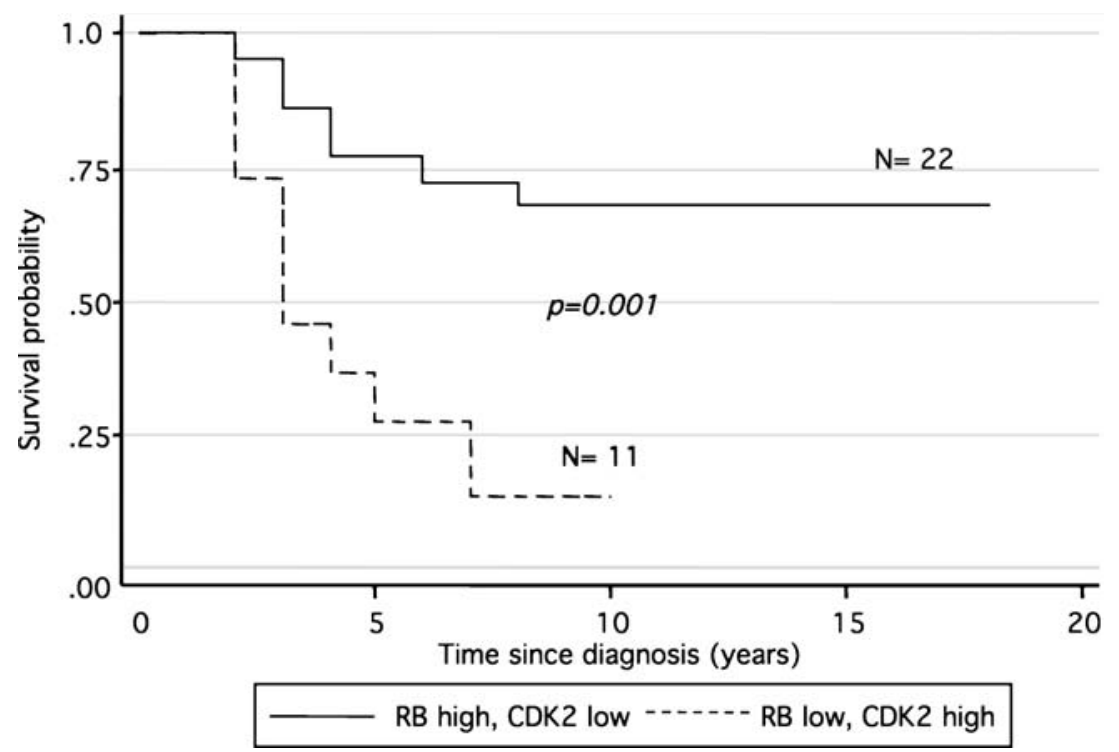

whilst AKT1 and AKT3 ablation seem to inhibit the development of mammary adenocarcinoma in mice [13] suggesting a complex functional pattern for this group of proteins.

RAF-1 is a protein serine-threonine kinase that plays an important role in cell growth, proliferation and survival. Our results show that patients with high RAF-1 status had a decreased disease survival as detected by Cox multivariate analysis (Fig. 7). These data agree with previous findings by Mewani et al. [15], these authors reported that an overexpression of RAF-1 in mammary epithelial cells induced the expression of genes promoting cell proliferation, invasiveness, angiogenesis, cell survival and also with mitogen-activated protein kinase kinase/extracellular regulated kinase-independent functions [14].
It is well-documented that the RB pathway plays a critical role in cell cycle progression, acting as a crucial negative regulator of cellular proliferation [23]. Our latest results on $\mathrm{RB}$ expression level and survival indicate a protective role of this molecule in breast cancer progression; patients with high RB seem to have a longer survival time (Fig. 3). Our findings agree with Derenzini et al. [4], who found that patients with low RB mRNA and a high level of phosphorylated RB labelling index had shorter disease-free intervals and overall survival time. Moreover, our data show that the CDK2 and RB expression levels, when combined together, are well able to predict patients' survival. The fact that patients characterised by a high status of CDK2 and a low status of RB presented a worse prognosis agrees with the role of these molecules in
Fig. 9 Kaplan-Meier survival curves for patients with respect to Cytokeratin 8 and HER2 expression level in the subgroups of high CK8 and low HER2 or low CK8 and high HER2 status

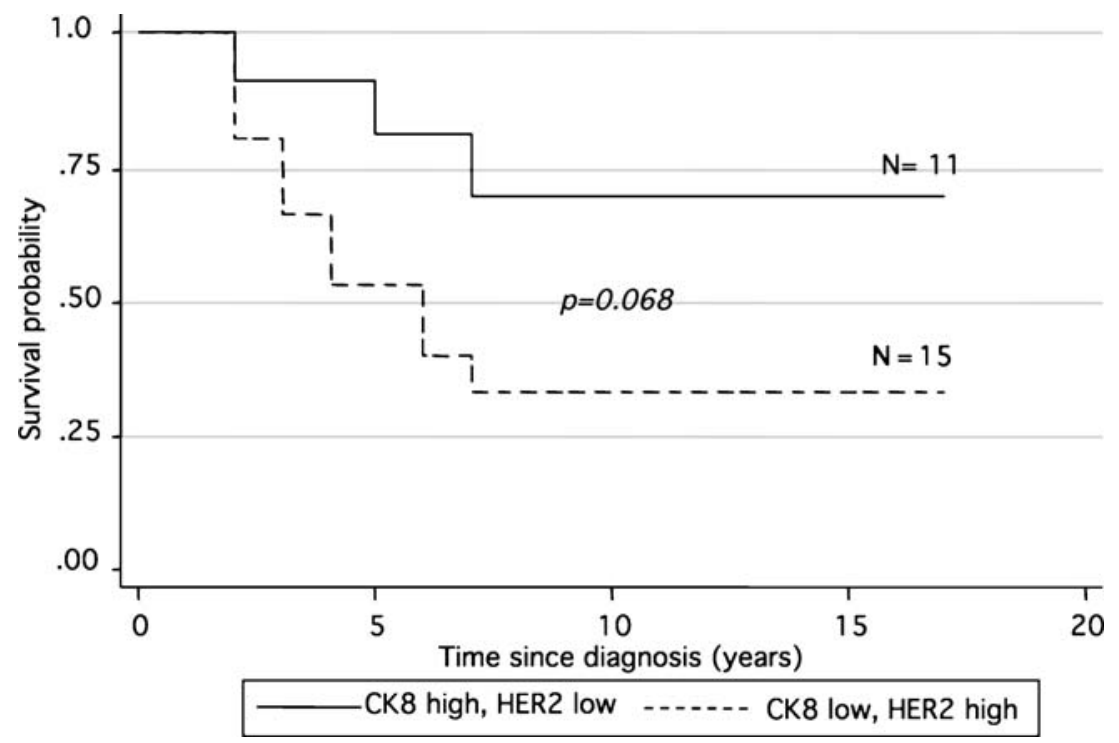


progression of the cell cycle through the G1/S phase and even indicated overcoming of this control checkpoint.

CK8 is characteristically present in luminal cells of a normal mammary gland. A wide range of cytokeratin expression is often seen in invasive breast cancer, which may be caused by loss of differentiation of the tumour cells [28]. In our study, higher expression levels of CK8 were associated in Cox multivariate analysis with better prognosis, and CK8 has a protective influence on patients' survival. Our data are concordant with the investigation by Fuchs et al. [9] based on IHC in breast carcinomas and breast cancer cell lines. That study revealed that suppression of CK8 was significantly correlated with short survival. Moreover, in cell lines with increasing invasion potential, epithelial keratins were also lost [9]. In our case study, CK8 (at high-expression status) in association with HER2 (low-expression status) identifies a group of patients with better prognosis. In this group of patients, the oestrogen receptor (ER) status was analysed by RT-PCR as previously reported [16] (data not shown). The patients of the group characterised by a high expression level of CK8 and low expression levels of HER2 were all positive for ER at the mRNA level. On the contrary, the other group, defined by the opposite pattern of the two markers (high HER2 and low CK8 levels), presented very low or no expression levels of the ER. These data agree with the recently reported molecular classification of breast cancer [20]. Patients with negative or weakly positive ER status and higher expression levels of HER2 presented a worse outcome, in accordance with Perreard et al. [20] who found ER-negative tumours more aggressive.

Of the 22 markers analysed in our study, 15 genes did not show influence on patients' survival. Among them, telomerase components need a comment. This enzyme catalyses the de novo synthesis of telomere repeats, which is necessary for unlimited cellular proliferation. The telomerase reverse transcriptase hTRT was detected at mRNA levels both in normal breast and breast cancer tissues [11]. Recently, Salhab et al. [22] investigated the expression level of the key telomere-related genes in human breast cancer and found that hTRT levels predicted the patients' overall survival. In our study, we did not find any association among telomerase components (hTR, the RNA template, and hTRT, the catalytic component), pathological parameters and patients' survival. One possible explanation is the normalisation methodology used by Salhab. Indeed, the authors normalised the expression levels of the telomerase genes against cytokeratin 19 (CK19) and not against one or more reliable housekeeping genes. This could modify the expression level of the telomerase machinery according to the level of CK19 expressed in cancer epithelial cells. From our study, it seems that telomerase expression could be a prerequisite for the early phase of tumorigenesis rather than a mechanism connected with progression. The presence and relative abundance of epithelial cells expressing telomerase within normal breast tissues could suggest that breast tumours arise from these highly expressing telomerase epithelial cells [11].

The molecular tumour progression analysed in the present study shows the natural history of the disease because the enrolled patients were only treated with the same type of surgical therapy. Our findings strongly demonstrate that the combination of two pairs of functionally conflicting markers (RB and CDK2 on one side, CK8 and HER2 on the other) may be divided into sub-groups that cover more than $50 \%$ of the patients, and these pairs of markers are able to identify sub-groups of patients with shorter relapse-free and survival times from those with long survival without recurrences. Groups characterised by low RB and high CDK2 (27\% of survivors at 5 years and $14 \%$ at 10 years) as with low CK8 and high HER2 (53\% of survivors at 5 years and $33 \%$ at 10 years) have a higher risk of early recurrence and death, suggesting that breast cancer progression in these patients could be related to these markers. Moreover, the recognition of these sub-groups of patients with higher risk of early relapse could have further relevance for the selection of patients to submit to therapy against HER2 or also against CDK2 as possible therapy targets. On the contrary, those with low CDK2 expression and high RB ( $68 \%$ of survival after 15 years) and those with high CK8 and low HER2 (69\% of survival after 15 years) present a very good prognosis.

These results are preliminary because the real predictive value of these double-biomarker groups could result only from larger retrospective and prospectively randomised studies to evaluate that the use of these molecular assays yield better clinical outcome than current clinical decisionmaking systems. Moreover, a possible use of IHC markers as surrogates for the reported genes could represent a further simplification with the prospective of introducing them into routine tumour management.

\section{References}

1. Agenzia Regionale della Sanità (2002) Registro tumori regionaledati di incidenza 1995-1998. Agenzia Regionale della Sanità, Italy

2. Bonin S, Brunetti D, Benedetti E, Gorji N, Stanta G (2006) Expression of cyclin-dependent kinases and CDC25a phosphatase is related with recurrences and survival in women with peri- and post-menopausal breast cancer. Virchows Arch 448(5):539-544

3. Bonin S, Pascolo L, Croce LS, Stanta G, Tiribelli C (2002) Gene expression of $\mathrm{ABC}$ proteins in hepatocellular carcinoma, perineoplastic tissue, and liver diseases. Mol Med 8(6):318-325

4. Derenzini M, Montanaro L, Vici M, Barbieri S, Ceccarelli C, Santini D, Taffurelli M, Martinelli GN, Trere D (2007) Relationship between the RB1 mRNA level and the expression of phosphorylated RB protein in human breast cancers: their relevance in cell proliferation 
activity and patient clinical outcome. Histol Histopathol 22(5):505513

5. Dimri GP (2005) What has senescence got to do with cancer? Cancer Cell 7(6):505-512

6. Elston CW, Ellis IO (1991) Pathological prognostic factors in breast cancer. I. The value of histological grade in breast cancer: experience from a large study with long-term follow-up. Histopathology 19 (5):403-410

7. Fan C, Oh DS, Wessels L, Weigelt B, Nuyten DS, Nobel AB, van, $t$ Veer LJ, Perou CM (2006) Concordance among gene-expressionbased predictors for breast cancer. N Engl J Med 355(6):560-569

8. Fresno Vara JA, Casado E, de Castro J, Cejas P, Belda-Iniesta C, Gonzalez-Baron M (2004) PI3K/Akt signalling pathway and cancer. Cancer Treat Rev 30(2):193-204

9. Fuchs IB, Lichtenegger W, Buehler H, Henrich W, Stein H, Kleine-Tebbe A, Schaller G (2002) The prognostic significance of epithelial-mesenchymal transition in breast cancer. Anticancer Res 22(6A):3415-3419

10. Greene FL, Page DL, Fleming ID, Fritz A, Balch MC, Haller DG, Morrow M (2002) AJCC cancer staging manual. Springer, New York

11. Hines WC, Fajardo AM, Joste NE, Bisoffi M, Griffith JK (2005) Quantitative and spatial measurements of telomerase reverse transcriptase expression within normal and malignant human breast tissues. Mol Cancer Res 3(9):503-509

12. Kendall SD, Linardic CM, Adam SJ, Counter CM (2005) A network of genetic events sufficient to convert normal human cells to a tumorigenic state. Cancer Res 65(21):9824-9828

13. Maroulakou IG, Oemler W, Naber SP, Tsichlis PN (2007) Akt1 ablation inhibits, whereas Akt2 ablation accelerates, the development of mammary adenocarcinomas in mouse mammary tumor virus (MMTV)-ErbB2/neu and MMTV-polyoma middle $\mathrm{T}$ transgenic mice. Cancer Res 67(1):167-177

14. McCubrey JA, Steelman LS, Abrams SL, Lee JT, Chang F, Bertrand FE, Navolanic PM, Terrian DM, Franklin RA, D'Assoro AB, Salisbury JL, Mazzarino MC, Stivala F, Libra M (2006) Roles of the RAF/MEK/ERK and PI3K/PTEN/AKT pathways in malignant transformation and drug resistance. Adv Enzyme Regul 46:249-279

15. Mewani RR, Tian S, Li B, Danner MT, Carr TD, Lee S, Rahman A, Kasid UN, Jung M, Dritschilo A, Gokhale PC (2006) Gene expression profile by inhibiting Raf-1 protein kinase in breast cancer cells. Int J Mol Med 17(3):457-463

16. Nardon E, Buda I, Stanta G, Buratti E, Fonda M, Cattin L (2003) Insulin-like growth factor system gene expression in women with type 2 diabetes and breast cancer. J Clin Pathol 56(8):599-604
17. Paredes J, Lopes N, Milanezi F, Schmitt FC (2007) P-cadherin and cytokeratin 5: useful adjunct markers to distinguish basal-like ductal carcinomas in situ. Virchows Archiv 450:73-80

18. Parkin DM, Whelan SL, Ferlay J, Raymond L, Young J (1997) Cancer incidence in five continents, vol. VII. International Agency for Research on Cancer, Lyon, pp 562-565

19. Penland SK, Keku TO, Torrice C, He X, Krishnamurthy J, Hoadley KA, Woosley JT, Thomas NE, Perou CM, Sandler RS, Sharpless NE (2007) RNA expression analysis of formalin-fixed paraffin-embedded tumors. Lab Invest 87(4):383-391

20. Perreard L, Fan C, Quackenbush JF, Mullins M, Gauthier NP, Nelson E, Mone M, Hansen H, Buys SS, Rasmussen K, Orrico AR, Dreher D, Walters R, Parker J, Hu Z, He X, Palazzo JP, Olopade OI, Szabo A, Perou CM, Bernard PS (2006) Classification and risk stratification of invasive breast carcinomas using a real-time quantitative RT-PCR assay. Breast Cancer Res 8(2):R23

21. Pusztai L, Mazouni C, Anderson K, Wu Y, Symmans WF (2006) Molecular classification of breast cancer: limitations and potential. Oncologist 11(8):868-877

22. Salhab M, Jiang WG, Newbold RF, Mokbel K (2007) The expression of gene transcripts of telomere-associated genes in human breast cancer: correlation with clinico-pathological parameters and clinical outcome. Breast Cancer Res Treat (in press)

23. Sherr CJ, McCormick F (2002) The RB and p53 pathways in cancer. Cancer Cell 2(2):103-112

24. Stanta G, Bonin S (1998) RNA quantitative analysis from fixed and paraffin-embedded tissues: membrane hybridization and capillary electrophoresis. Biotechniques 24(2):271-276

25. Stanta G, Bonin S, Losi L, Eusebi V (1998) Molecular characterization of intraductal breast carcinomas. Virchows Arch 432(2):107-111

26. Stanta G, Bonin S, Niccolini B, Raccanelli A, Baralle F (1999) Catalytic subunit of telomerase expression is related to RNA component expression. FEBS Lett 460(2):285-288

27. Stanta G, Schneider C (1991) RNA extracted from paraffinembedded human tissues is amenable to analysis by PCR amplification. Biotechniques 11(3):304, 306, 308

28. Su L, Morgan PR, Lane EB (1996) Expression of cytokeratin messenger RNA versus protein in the normal mammary gland and in breast cancer. Human Pathol 27(8):800-806

29. Tokunaga E, Kimura Y, Mashino K, Oki E, Kataoka A, Ohno S, Morita M, Kakeji Y, Baba H, Maehara Y (2006) Activation of $\mathrm{PI} 3 \mathrm{~K} / \mathrm{Akt}$ signaling and hormone resistance in breast cancer. Breast Cancer 13(2):137-144

30. Yankaskas BC (2005) Epidemiology of breast cancer in young women. Breast Dis 23:3-8 